\title{
Die Coronavirus-Krise gemeinsam durchstehen
}

\section{Samia Hurst}

Institut Ethique Histoire Humanités (iEH2), Medizinische Fakultät, Genf

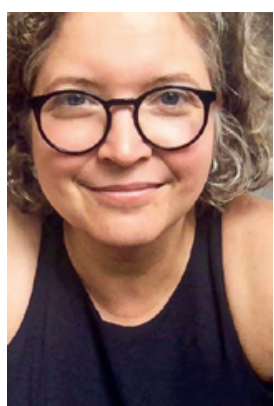

Während ich diese Zeilen schreibe, breitet sich die Coronavirus-Epidemie in der Schweiz aus. Mit dieser Epidemie gehen Massnahmen zu ihrer Eindämmung einher. Und das mit enormer Geschwindigkeit. Im Spannungsfeld zwischen Verharmlosung und Panik kommen immer mehr Fragen auf. Nehmen wir uns also die Zeit einer kurzen Kaffeepause für ein paar Gedanken. In einer Zeit, in der die Angst vor Infektionskrankheiten wieder aufflammt, verdienen einige ethische Fragen eine genauere Betrachtung.

Zunächst einmal retten einfache Massnahmen - so simpel, dass sie lächerlich erscheinen mögen - tatsächlich Leben. Vorsicht ist in diesen Zeiten also moralische Pflicht. Zwar können wir die Epidemie nicht mehr abwenden. Aber wir können ihren Verlauf abflachen und so die Zahl der Opfer begrenzen. Wenn die Epidemie langsamer verläuft, können unsere Spitäler mehr Patientinnen und Patienten medizinisch optimal versorgen und die Mortalität senken. Die meisten von uns hatten noch nie so viele Leben buchstäblich in der Hand.

Hinzu kommt, dass bei Massnahmen zum Schutz der öffentlichen Gesundheit immer eine Abwägung stattfindet. Auf dem Kreuzfahrtschiff Diamond Princess, das in einem japanischen Hafen festlag, kamen die falschen Massnahmen zum Einsatz. Mit dem Ergebnis, dass jeder fünfte Passagier erkrankte. In Wuhan, wo die chinesische Staatsmacht brutal eingriff, erkrankte weniger als einer von hundert Menschen. Zwischen diesen beiden Extremen liegt der Handlungsspielraum unserer Entscheidungsträgerinnen und Entscheidungsträger. Wie viele Rechte müssen - wenn auch nur vorübergehend geopfert werden, um wie viele Menschenleben zu retten? Es wird unmöglich sein, das absolut Richtige zu tun. Bringen wir es auf den Punkt: Es geht darum, die am wenigsten schlimme Lösung zu finden.

Es gibt gewisse Aspekte, die die Verhängung einer Ausgangssperre rechtfertigen. Unsere kanadischen Kolleginnen und Kollegen haben während des SARSAusbruchs 2003 entsprechende Kriterien formuliert. Die Massnahme muss zielgerichtet sein. Die Massnahme sollte gerade so einschränkend wie nötig sein, um noch die erhoffte Wirkung zu erzielen. Sie muss verhältnismässig und den Einsatz wert sein. Sie muss transparent sein: Wir müssen wissen, welche Massnahme von wem und aus welchem Grund beschlossen wird. Und für diejenigen, die das Haus nicht verlassen dürfen, muss es eine Möglichkeit geben, eine solche Entscheidung anzufechten. Epidemien können eine zeitweilige Einschränkung unserer Rechte bedeuten, doch müssen wir vor Willkür geschützt sein. Je nachdem, wie viele schwere Erkrankungen gleichzeitig auftreten, muss in den Spitälern eine Triage erfolgen, so wie im Katastrophenfall üblich. Wir müssen alle behandeln. Aber in einer solchen Situation können die Spitäler nicht alle Kranken aufnehmen, ebenso wenig wie in den Intensivstationen dann für alle schweren Fälle Kapazitäten vorhanden sein werden. Durch die Triage wird all denjenigen die Aufnahme verweigert, die entweder «nicht krank genug» oder aber «zu krank» sind, weil ihre Überlebenschancen auch bei einer Intensivbehandlung zu gering wären. Diese Einschätzung muss transparent sein und auf dem Grundsatz beruhen, dass jedes Menschenleben gleich viel wert ist. Es kann nicht angehen, dass Menschen abgewiesen werden, weil sie als "weniger wichtig» gelten. Ohne eindeutige Gleichbehandlung wäre eine solche Massnahme in kurzer Zeit nicht mehr umsetzbar [1].

Aber vielleicht ist der wichtigste Punkt, den wir beachten müssen, der scheinbar unauffälligste. In einer Zeit, in der wir körperlich Abstand halten müssen, gilt es die soziale Nähe umso mehr zu pflegen. Ob wir uns nun aus zwei Meter Entfernung oder per Video unterhalten: Wir sind weiter aufeinander angewiesen. Nur gemeinsam werden wir diese schwierige Zeit durchstehen. Unsere Institutionen werden unter Beweis stellen, was sie am besten können; es wird aber auch zutage treten, wo ihre Schwächen liegen. Durch unser tägliches Handeln können wir beeinflussen, wohin diese Krankheit führt. Indem wir auf den Handschlag verzichten, nicht aber auf unser Lächeln. Nach der Epidemie werden wir weiter zusammenleben. Und wir werden uns daran erinnern, wie wir uns in dieser schwierigen Zeit verhalten haben

\section{Literatur}

1 Siehe hierzu die Richtlinien der Schweizerischen Akademie der Medizinischen Wissenschaften: samw.ch/de/corona. 